\title{
Empowering and Engaging Solutions for Operator 4.0 - Acceptance and Foreseen Impacts by Factory Workers
}

\author{
Eija Kaasinen ${ }^{1(\bowtie)}$, Susanna Aromaa ${ }^{1}$, Päivi Heikkilä ${ }^{1}$, \\ and Marja Liinasuo ${ }^{2}$ \\ 1 VTT Technical Research Centre of Finland Ltd., Tampere, Finland \\ \{eija.kaasinen, susanna.aromaa, paivi.heikkila\}@vtt.fi \\ ${ }^{2}$ VTT Technical Research Centre of Finland Ltd., \\ P.O. Box 1000, 02044 Espoo, Finland \\ marja.liinasuo@vtt.fi
}

\begin{abstract}
Industry 4.0 factories require smart and skilled operators - Operators 4.0. We evaluated three Operator 4.0 solutions that aim to empower and engage workers, so that workers can understand and develop their own competences and they can take an active role in developing the manufacturing environment. 118 shop stewards from different factories assessed user experience, user acceptance and foreseen impacts of the solutions. The results show that there are high expectations towards the virtual factory-based participatory design solution. Opinions were shared about empowering the worker by feedback about worker well-being and work achievements. The solution was seen interesting and useful but there were doubts regarding privacy. Contextual knowledge sharing was felt important but the workers were a bit skeptical as many previous knowledge sharing attempts had been failed. The results give insight to how the solutions should be further developed and how the solutions should be introduced at factory floor.
\end{abstract}

Keywords: Operator $4.0 \cdot$ User study $\cdot$ User experience $\cdot$ User acceptance $\cdot$ Impact assessment

\section{Introduction}

A central component of Industry 4.0 is its human-centricity, described as development towards the Operator 4.0 concept [1]. Operator 4.0 refers to smart and skilled operators of the future, who will be assisted by automated systems allowing the operators to utilize and develop their creative, innovative and improvisational skills, without compromising production objectives [1]. In future industrial environments, workers are expected to act as strategic decision-makers and flexible problem-solvers [2]. Romero et al. [1] postulated an Operator 4.0 typology with eight different operator types such as Healthy Operator, Collaborative Operator and Social Operator. In later papers, Romero et al. further defined the concepts of Healthy Operator [3] and Social Operator [4]. The visionary papers introduce future concepts but not yet evaluation results. 
Cognitive workload can be relieved by assessing the operator's well-being at work by using wearable technology [3]. With the advances in personal, wearable health technologies, employees can get empowering feedback on their well-being [5]. Virtual reality (VR) technologies are valid tools to support participatory design (PD), because they support common understanding and collaboration among designers and users [6]. To improve team performance, organizations must ensure that knowledge is both shared and applied [7]. New technologies can encourage collaboration among workers, support problem-solving and bring together the right people [4]. The Operator 4.0 paradigm shift cannot succeed simply by introducing new technologies to the factory floor, but work processes need to be reshaped in parallel [8].

The aim of the study described in this paper was to evaluate user experience, user acceptance and foreseen impacts of three Operator 4.0 solutions developed in the Factory2Fit project (www.factory2fit.eu): Worker Feedback Dashboard, Participatory Design with a Virtual Factory and Contextual Knowledge Sharing. The solutions can be seen as examples of Operator 4.0 types Healthy Operator, Virtual Operator and Social Operator as defined by Romero et al. $[1,3,4]$. The leading idea of the solutions is that the workers are the best experts of their own work and they should be given effective possibilities to develop their competence, based on their own initiative, and their role should be more active in designing and developing manufacturing processes [8].

In this paper we describe the three Operator 4.0 solutions in Sect. 2. In Sect. 3 we describe the methods, in Sect. 4 the results and in Sect. 5 conclusions.

\section{The Empowering and Engaging Operator 4.0 Solutions}

In the following, we describe the three solutions that we assessed with factory workers.
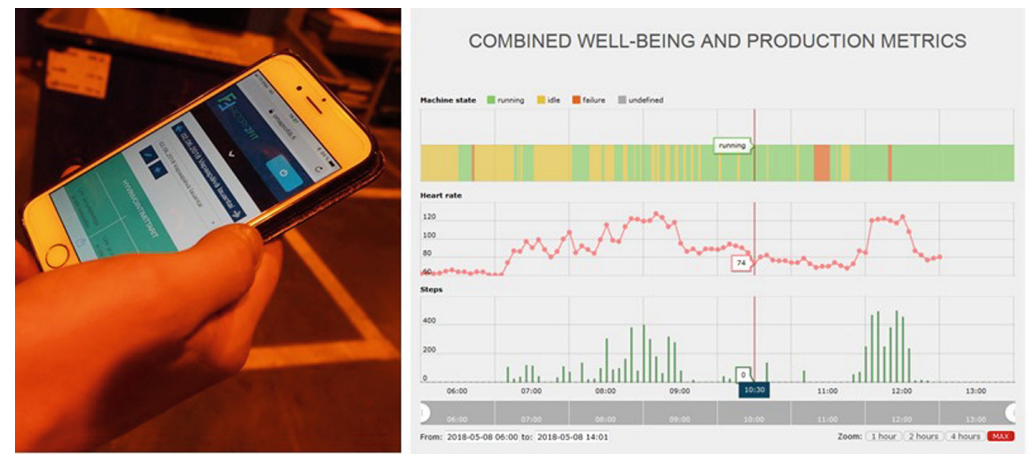

Fig. 1. Worker Feedback Dashboard.

Worker Feedback Dashboard (Fig. 1) is a web-based solution that provides datadriven, personal feedback to workers about their well-being and work performance [9]. It highlights the accomplishments of the day, creates awareness of the possible relations between selected well-being and production measures and demonstrates progress and 
development at work. The solution shows both visualized work shift-specific data as well as longer-term trends. The feedback is generated mainly based on automatically collected data: well-being metrics from a personal well-being tracker, such as an activity wristband and work performance metrics from the factory production system.

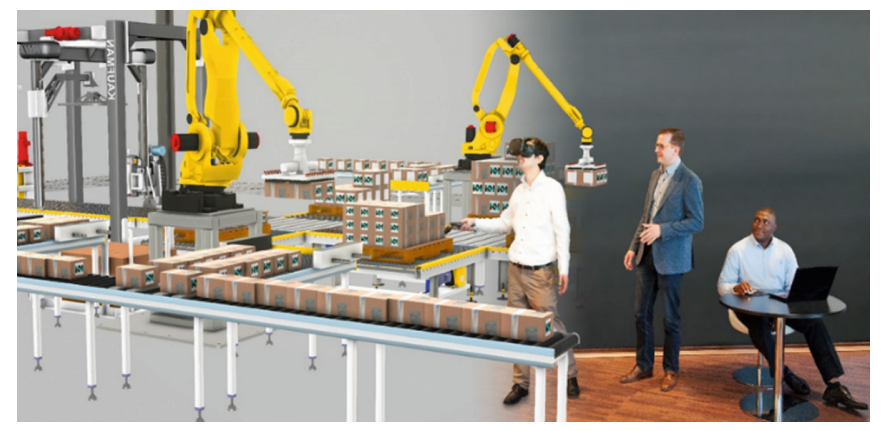

Fig. 2. Participatory design with a virtual factory.

Participatory Design with a Virtual Factory supports co-design of factory work with all stakeholders, such as workers, supervisors, management and R\&D [10]. A 3D simulation and visualization platform (Visual Components 4.1) is used as the virtual factory platform. It allows the creation of virtual factory layouts at different levels of detail from a simple machine to the entire factory floor (Fig. 2). The virtual factory supports gaining a shared understanding of the design and sharing knowledge. During a participatory design session, the virtual factory can be viewed via a projector, or it can be experienced more realistically with a head-mounted display (Fig. 2).

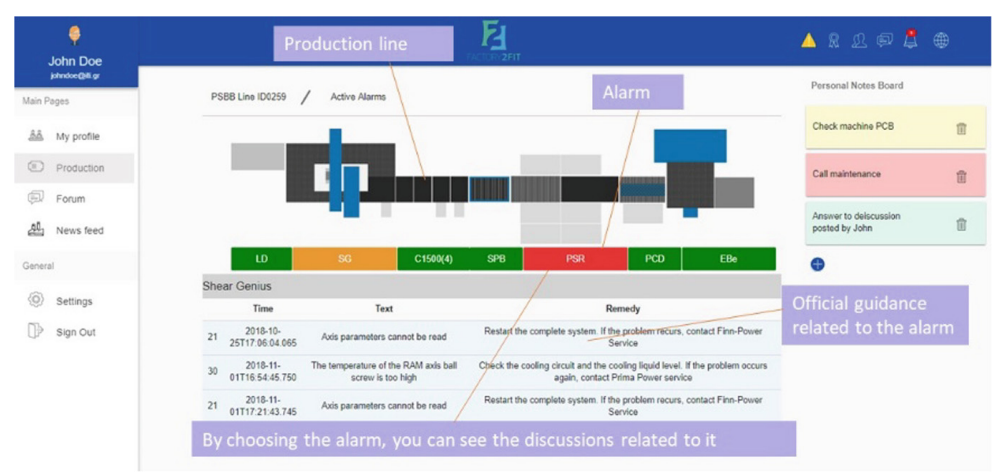

Fig. 3. Contextual Knowledge Sharing integrated into the user interface of a production line. 
Contextual Knowledge Sharing is a platform for work-related discussions, connected to the physical work context such as work machines [10]. The objective of Contextual Knowledge Sharing is to support collaboration, problem solving and knowledge sharing among workers. The solution can be used in one factory or the discussion can be extended to several factories with similar productions. Contextual Knowledge Sharing can be used with a computer or mobile device. It is preferably integrated into other factory information systems (Fig. 3), so that discussions are connected to actual machines and situations on the factory floor.

\section{Methods}

The Industrial Union is the largest trade union in Finland representing workers in the metals, manufacturing and paper industries. Factory2Fit solutions were presented to the representatives of the Industrial Union, who were in charge of organizing their annual meeting. They chose three Operator 4.0 solutions for the evaluation, based on their relevance to the annual meeting participants. Sharing interesting knowledge was important to ensure mutual benefit for both the participants and the Factory2Fit project.

During the Industrial Union's annual meeting, three workshops were organized, one for each Operator 4.0 solution to be assessed. The workshops were repeated nine times, each lasting for $20 \mathrm{~min}$ with $10-15$ participants. The participant groups remained the same in each workshop but the order of the workshops varied between the groups. In total, 118 shop stewards participated in the workshops. They all filled in a questionnaire that was based on Work Well-being Design and Evaluation Framework [11] and studied user experience, user acceptance and foreseen impacts on work satisfaction and productivity. Each workshop had the following agenda: (1) workshop host presented the solution with slides and video demos; (2) the participants discussed the solution, and (3) the participants filled in the questionnaire.

$21 \%$ of the participants were female and $79 \%$ male. All the participants were working in industry: $31 \%$ in large enterprises, $52 \%$ in medium-size enterprises and $17 \%$ in small companies. $72 \%$ of the participants were $40-59$ years old, $20 \%$ under 40 and $8 \%$ were over 60 . The participants were all shop stewards, so in that sense they were quite a homogenous group. However, as they came from different sized enterprises, there were differences especially in the degree of digitalization at their workplace.

\section{Results}

Figure 4 illustrates the user experience of the solutions. Participatory design had the best user experience but also the two other concepts had mainly positive user experiences. 


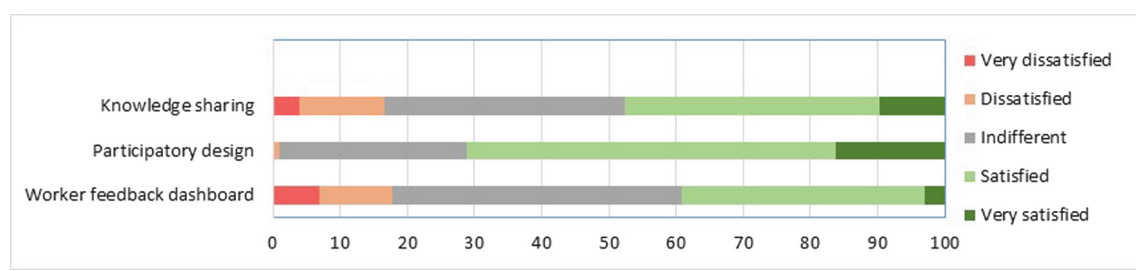

Fig. 4. User experience of the solutions.

\subsection{Worker Feedback Dashboard}

The Worker Feedback Dashboard clearly divided the opinions of the respondents (Fig. 5). $41 \%$ of the respondents considered the solution suitable for factory work while $21 \%$ responded that it would not fit factory work. $37 \%$ of the respondents were willing to use the solution themselves while $34 \%$ were not interested in using the solution in their work. About half of the respondents neither agreed nor disagreed on whether using the solution would improve productivity or work satisfaction. Positive impacts on productivity were expected by $20 \%$ of the participants, and positive impacts on work satisfaction by $23 \%$ of the participants.

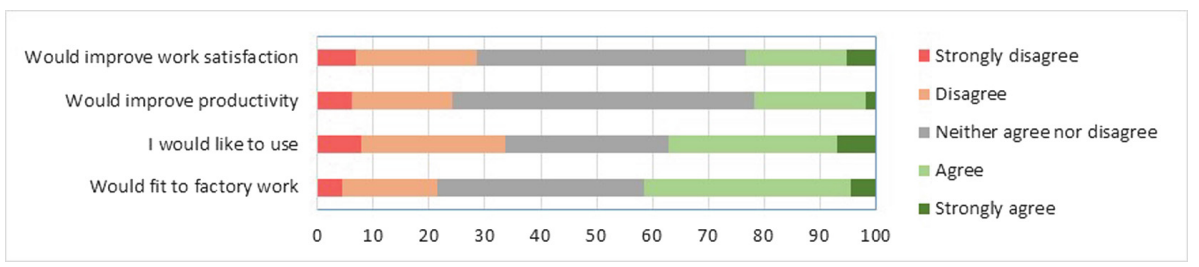

Fig. 5. Results of the Worker Feedback Dashboard assessment.

In most of the workshops, the Worker Feedback Dashboard solution raised several questions and vivid discussion. The participants asked questions related to the properties of the solution (e.g., what can be quantified) and the details and usefulness of the measurements. In some groups, the discussion was mainly neutral or positive, while in other groups the discussion focused on the potential risks of the solution. 49 respondents gave free feedback about the solution on the questionnaire. The main concerns were related to data security and privacy, and the participants were skeptical of whether the personal data would stay non-accessible to the employer.

\subsection{Participatory Design with a Virtual Factory}

The Participatory Design with a Virtual Factory was well accepted and positive impacts were foreseen (Fig. 6). 77\% of the participants agreed that this kind of solution would fit to factory work. $62 \%$ of the participants agreed that they would like to use this kind of solution in their work. Most participants agreed that the solutions would improve productivity (76\%) and work satisfaction (66\%). 


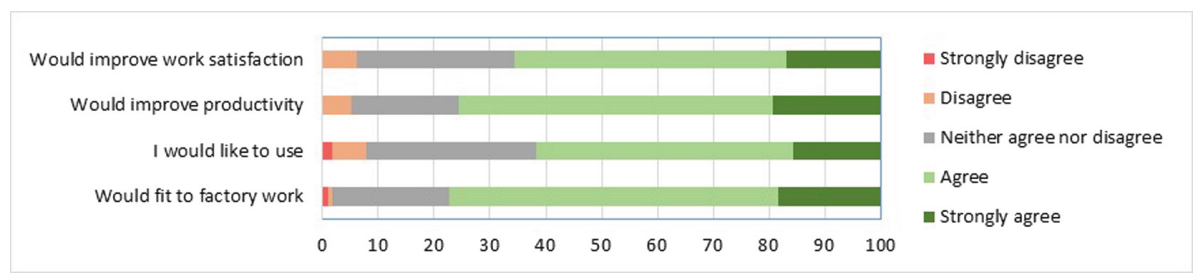

Fig. 6. Results of the Participatory Design with Virtual Factory assessment.

The participants provided feedback both on the questionnaire and during the discussions. 53 participants provided comments on the questionnaire. For some participants, the solution was quite new, whereas others had already been using similar solutions at work. The participants agreed that adopting the solution is already topical or will be in the near future. The participants liked the idea that workers are able to participate in the design of their own work but were concerned that employers would not listen to them. The participants commented that the solution could decrease time and costs during layout changes by instantly designing good layouts. With welldesigned production lines, productivity will increase. Some participants were concerned that if only productivity and efficiency are improved, there would be no positive impact on work satisfaction.

\subsection{Contextual Knowledge Sharing}

With Contextual Knowledge Sharing, results were distributed (Fig. 7) but so that positive responses outnumbered the negative ones and the moderately positive attitude towards the knowledge sharing platform was the most dominant. As much as $50 \%$ of the respondents considered the solution suitable for factory work and, furthermore, the majority of respondents $(56 \%)$ thought that the solution would improve work productivity. However, less than half of respondents $(44 \%)$ were willing to use the solution themselves and only $39 \%$ assumed the solution would improve work satisfaction.

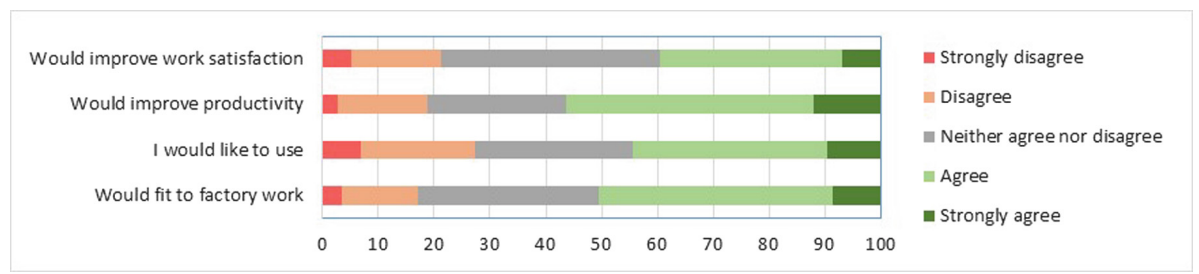

Fig. 7. Results of the knowledge sharing solution assessment.

Even if the feedback on the questionnaires was mainly positive, the discussion and the comments tended to focus on foreseen problems and concerns. 57 participants 
provided comments on the questionnaire and discussion was always raised during the solution presentation. Respondents suggested suitable usage contexts such as maintenance and problem-solving. The solution was not found appropriate when, e.g., a phone is not allowed at a workplace or when work is highly manual. Some participants did not fully understand the contextuality of the solution and claimed that similar solutions exist already. There were concerns that the usage will require too much time or attention at work, causing various types of risks; or that people are not willing or able to share their knowledge, especially in written form.

\section{Conclusions}

The feedback was mainly positive but there were also concerns, especially related to the Worker Feedback Dashboard and Contextual Knowledge Sharing. Participatory Design with a Virtual Factory was clearly the most accepted solution.

The Worker Feedback Dashboard divided opinions. These divided opinions and concerns should be considered when introducing the Worker Feedback Dashboard to the workplace. Ethical aspects and the potential impacts on all stakeholders shall be considered in the design of the solution. For user acceptance, the workers should participate in planning how to utilize the solution, and what kind of work practices should be agreed related to its use. The results indicate that there are potential lead users for the Worker Feedback Dashboard. Introducing the solution via these lead users would facilitate showing the impacts and could then encourage those who were more doubtful to join. Still, using this kind of a solution should be voluntary.

Many participants commented that Participatory Design with a Virtual Factory solution is what they really should have at their workplace, and most participants expected positive impacts both on productivity and work satisfaction. The concerns were mainly related to the culture at the workplace - whether the suggestions of the workers would be considered.

Contextual Knowledge Sharing was quite well accepted but it was evident that there have been several attempts already at the workplaces to introduce tools for knowledge sharing. Attendees seemed a bit fed-up with "again another knowledgesharing tool". During the short presentation of the solution, the participants did not fully understand the novelty value of the solution in connecting the discussions to the physical factory floor context. Still, $55 \%$ of the participants expected positive impacts on productivity and $39 \%$ on work satisfaction. When developing the concept further, the connection to the work context has to be emphasized better. Knowledge sharing should be fully integrated with production systems, extending their content with the user-generated discussion content. For a quick start, knowledge-sharing should be based on actual user communities that already know and trust each other.

The study described in this paper was conducted with a relatively large, homogenous group of participants, as all of them were shop stewards in factories. However, there are some limitations in the results. The solutions were presented to the participants quite briefly, and they could not try out the solutions themselves. The participants filled in the questionnaire after the group discussion, so it is possible that 
the discussion affected their opinion. However, the discussions may also have supported understanding the proposed solutions better.

Overall, the results are quite promising and encourage introducing Operator 4.0 solutions to factory work. The results of the assessment will be useful in further developing the solutions as well as in designing how to introduce them on the factory floor. Assessing not only immediate implications but also foreseen impacts with the factory workers raised important issues in the discussions and in the comments. These kinds of discussions will be important also in connection to local factory floor design of the solutions and their adoption.

Acknowledgements. This research was funded under the European Commission's H2020 framework programme in the project Factory2Fit "Empowering and Participatory Adaptation of Factory Automation to Fit for Workers" (Grant agreement 723277). The authors are grateful to all the researchers and company representatives who contributed to and supported the work presented in this publication. Special thanks to the representatives of the Finnish Industrial Union for their good collaboration.

\section{References}

1. Romero, D., et al.: Towards an operator 4.0 typology: a human-centric perspective on the fourth industrial revolution technologies. In: International Conference on Computers \& Industrial Engineering (CIE46), pp. 1-11 (2016)

2. Gorecky, D., Schmitt, M., Loskyll, M., Zühlke, D.: Human-machine-interaction in the industry 4.0 era. In: 12th IEEE International Conference on Industrial Informatics (INDIN), pp. 289-294. IEEE (2014)

3. Romero, D., Mattsson, S., Fast-Berglund, Å., Wuest, T., Gorecky, D., Stahre, J.: Digitalizing occupational health, safety and productivity for the operator 4.0. In: Moon, I., Lee, G.M., Park, J., Kiritsis, D., von Cieminski, G. (eds.) APMS 2018. IAICT, vol. 536, pp. 473-481. Springer, Cham (2018). https://doi.org/10.1007/978-3-319-99707-0_59

4. Romero, D., Wuest, T., Stahre, J., Gorecky, D.: Social factory architecture: social networking services and production scenarios through the social internet of things, services and people for the social operator 4.0. In: Lödding, H., Riedel, R., Thoben, K.-D., von Cieminski, G., Kiritsis, D. (eds.) APMS 2017. IAICT, vol. 513, pp. 265-273. Springer, Cham (2017). https://doi.org/10.1007/978-3-319-66923-6_31

5. Mattila, E., Orsama, A.L., Ahtinen, A., Hopsu, L., Leino, T., Korhonen, I.: Personal health technologies in employee health promotion: usage activity, usefulness, and health-related outcomes in a 1-year randomized controlled trial. JMIR Mhealth Uhealth 1(2), e16 (2013)

6. Bruno, F., Muzzupappa, M.: Product interface design: a participatory approach based on virtual reality. Int. J. Hum. Comput. Stud. 68(5), 254-269 (2010)

7. Choi, S.Y., Lee, H., Yoo, Y.: The impact of information technology and transactive memory systems on knowledge sharing, application, and team performance: a field study. MIS Q. 34 (4), 855-870 (2010)

8. Kaasinen, E., et al.: Empowering and engaging industrial workers with operator 4.0 solutions. Comput. Ind. Eng. (2019, in press)

9. Heikkilä, P., Honka, A., Kaasinen, E.: Quantified factory worker: designing a worker feedback dashboard. In: 10th Nordic Conference on Human-Computer Interaction, NordiCHI 2018, pp. 515-523. ACM, New York (2018) 
10. Aromaa, S., et al.: User evaluation of industry 4.0 concepts for worker engagement. In: Ahram, T., Karwowski, W., Taiar, R. (eds.) IHSED 2018. AISC, vol. 876, pp. 34-40. Springer, Cham (2019). https://doi.org/10.1007/978-3-030-02053-8_6

11. Kaasinen, E., et al.: A worker-centric design and evaluation framework for operator 4.0 solutions that support work well-being. In: Barricelli, B.R., et al. (eds.) HWID 2018. IAICT, vol. 544, pp. 263-282. Springer, Cham (2019). https://doi.org/10.1007/978-3-03005297-3_18

Open Access This chapter is licensed under the terms of the Creative Commons Attribution 4.0 International License (http://creativecommons.org/licenses/by/4.0/), which permits use, sharing, adaptation, distribution and reproduction in any medium or format, as long as you give appropriate credit to the original author(s) and the source, provide a link to the Creative Commons license and indicate if changes were made.

The images or other third party material in this chapter are included in the chapter's Creative Commons license, unless indicated otherwise in a credit line to the material. If material is not included in the chapter's Creative Commons license and your intended use is not permitted by statutory regulation or exceeds the permitted use, you will need to obtain permission directly from the copyright holder. 